\title{
An evaluation of photographic screening for neovascular age- related macular degeneration
}

DAL Maberley, C Isbister, P MacKenzie and

A Aralar

\begin{abstract}
Background Photographic screening for neovascular age-related macular degeneration (AMD) is not commonly employed because the prevalence of treatable disease is low and fluorescein angiography is considered necessary for the diagnosis of this form of AMD. However, there may be a role for colour retinal imaging in assisting with the diagnosis and triage of subjects with neovascular AMD. The purpose of this study was to evaluate the utility of colour fundus photographs for identifying subjects with potentially treatable neovascular AMD.
\end{abstract}

Methods A total of 74 stereo pairs of Kodachrome colour slides of subjects with AMD were evaluated (i) nonstereoscopically, (ii) stereoscopically, and (iii) stereoscopically with visual acuity and visual symptom data. Two retina specialists read the images to identify active exudative lesions.

Results The kappa statistic comparing the retinal specialists diagnosis of treatable neovascular AMD from color slides was excellent. The sensitivity and specificity of nonstereo images for the appropriate categorization of lesions was 0.95 and 0.90 respectively. The evaluation of stereo pairs was more sensitive, but less specific, $0.98,0.83$, as was the evaluation of stereo-pairs with clinical histories and visual acuities, 1.00, 0.77. Conclusions The evaluation of colour images for subjects with suspected exudative macular degeneration can be diagnostic for neovascular AMD and may expedite the appropriate referral of patients for more timely angiography and treatment. Incorporating more clinical information for the image evaluators ((i) stereo image pairs and/or (ii) presenting symptomatology and visual acuity data) led to a decrease in the false-negative rate, but also decreased the screening specificity.

Eye (2005) 19, 611-616. doi:10.1038/sj.eye.6701584 Published online 28 May 2004

Keywords: age-related macular degeneration; screening; sensitivity; specificity; interobserver agreement

With the refinement of imaging technologies and protocols for monitoring diabetic retinopathy, screening for this particular condition is now widely performed photographically. Conversely, screening for agerelated macular degeneration (AMD) is not common because the basic tenets associated with diseases that warrant screening are not frequently met for this condition.

Screening should focus on a serious disease that has an easily defined at-risk population. Evaluative testing needs to be reasonably noninvasive, cost-effective, and demonstrate a high degree of sensitivity and specificity. ${ }^{1}$ Once detected, the disease should have an available therapy that has been shown to reduce the risk of morbidity/mortality for those treated. Tests with a high sensitivity are better for disease identification and should lead to better patient outcomes. Tests with high specificities tend to lead to lower screening costs via limiting secondary resource utilization. These screening tenets are met for diabetic retinopathy ${ }^{2-9}$ ), but have not been thoroughly evaluated for AMD.

There are numerous reasons why neovascular AMD is not typically screened for. First, unlike diabetic retinopathy, high-risk individuals are identified primarily by retinal evaluation and not on the basis of an underlying systemic disease. Second, neovascular macular
Department of Ophthalmology University of British Columbia Vancouver BC, Canada

Correspondence: D Maberley 2550 Willow

St. Vancouver BC V5Z 3N9

Tel.: 6048754599

Fax: 6048754699

E-mail: dmaberle@

vanhosp.bc.ca

Received: 9 January 2004 Accepted: 15 March 2004 Published online: 28 May 2004

Financial support: None. 
degeneration tends to occur in the presence of new visual symptoms and, as such, often brings

itself to medical attention only after these develop.

Third, the treatment of neovascular (exudative)

macular degeneration requires an intravenous

fluorescein angiogram for lesion categorization and

measurement. Finally, the very narrow therapeutic

window through which exudative AMD passes also

makes screening for this form of macular degeneration

very difficult - given the low prevalence of active, early disease.

Until recently, the lack of successful treatments for AMD may also have limited interest in screening for this condition. With emerging therapies, macular degeneration has become a disease that would possibly benefit from a screening methodology. ${ }^{10,11}$ Presently, the definitive standard for the diagnosis of neovascular AMD is fluorescein angiography; however, this test is moderately expensive, only offered in larger centres, and is invasive. While colour stereoscopic images can be read to determine whether an individual will meet criteria for the initiation of antioxidant therapy, ${ }^{12}$ they have not been used to diagnose active neovascular AMD and to determine if an individual requires urgent flourescein angiography prior to photodynamic therapy or ablative photocoagulation. The rationale of screening for neovascular AMD is the assumption that facilitating access to ablative laser or photodynamic therapy earlier in the disease course will improve therapeutic outcomes. If successful, smaller and potentially less active lesions would be treated more frequently, resulting in more successful treatments as a whole. ${ }^{13}$

Community-based educational programmes may assist patients in seeking ophthalmic care earlier in the disease course of neovascular AMD; however, delays between the onset of symptoms and the patient's initial contact with an eye care provider are largely uncontrollable. The main 'controllable' delays in accessing timely care (after a patient presents to an eye care provider) typically arise during the process of obtaining a retinal opinion and/or fluorescein angiogram. A recent study found that $16 \%$ of patients with exudative macular degeneration were seen by retinal specialists more than 6 months after the onset of visual symptoms. ${ }^{14}$ Increasing the effectiveness of triage services through the use of photographic screening may improve the controllable components of the delay between first medical contact and treatment.

The current paper examines whether a photographic screening service might assist retinal specialists in providing a more timely diagnosis of neovascular AMD, facilitating timely angiographic evaluations and treatment. There is little information presently available regarding the usefulness of colour retinal images for the diagnosis and triage of wet forms of AMD.

\section{Methods}

After performing a power calculation as outlined below, angiography department records of the University of British Columbia/Vancouver General Hospital Eye Care Center were searched for a consecutive series of 40 subjects ( 80 eyes) who had been referred to the departmental retina service in the preceding 3 months (January 2002 to March 2002). Subjects were sent by general ophthalmologists with a diagnosis of 'age-related macular degeneration'.

For each individual, both eyes were imaged by colour fundus photography and fluorescein angiography. All retinal images were taken stereoscopically with a Zeiss FF-series $30^{\circ}$ fundus camera on Kodachrome slide transparency film with the images centred on the clinical macula. To maintain consistency with the AREDS photographic protocol, macular field 2 was used for grading. Photography was performed by certified retinal photographers using a standardized protocol. At separate reading sessions, all images were randomly presented to two retina specialists who were masked as to the status of the contralateral eye. The colour image readings were performed serially and independently by each specialist. First, the best-quality single image of the stereo pair was read. Second, the stereo-pair was evaluated. Third, the stereo pair was re-evaluated with knowledge of the subject's best-corrected visual acuity and their presenting complaint. The inclusion of a presenting complaint and best-corrected visual acuity for the affected eye (Snellen) was made done to provide information that a representative photographic screening program might collect during the evaluation of subjects in the field.

Readers were required to predict which colour images would demonstrate choroidal neovascularization and, hence, require a fluorescein angiogram. Readers were not asked to predict the underlying lesion composition or location, but to identify the presence of any form of choroidal neovascularization that might benefit from photocoagulation or photodynamic therapy. A simple yes or no response was recorded. For eyes that were deemed to need angiography, the readers were also asked to note which clinical features were present and which of these led to their recommendation for angiography.

Fluorescein angiograms taken at the same time as the colour images were read by the two retinal specialists at a separate (later) reading session to identify treatable lesions. A third retinal opinion was sought for grader 
disagreement on the angiographic interpretation. A treatable lesion was considered present if there was angiographic evidence of classic or occult choroidal neovascularization within field 2 . Isolated pigment epithelial detachments (serous, not fibrovascular) and areas of atrophic macular degeneration were not considered to require immediate angiography.

A power calculation was performed for two-sided tests comparing two binomial populations - assuming an alpha of 0.05 , a prevalence of wet macular degeneration of $50 \%$, and a clinical diagnostic difference of $25 \%$. For a sample of 80 images, the power to detect such a difference was $88 \%$. Once six images were excluded because of poor quality or the absence of stereo pairs, 74 eyes remained within the data set. Using these new parameters, the power calculation was re-run with a resultant power of $83 \%$.

Statistical analyses were performed with SPSS ${ }^{\mathrm{TM}}$ version 10.0 for PC (Chicago, IL, USA) for the primary analysis and StatXact ${ }^{\mathrm{TM}}$ software from Cytel (Cambridge, MA, USA) for contingency table analyses using exact test methodologies when cells were identified with $<5$ values. The University of British Columbia and Vancouver Hospital Ethics Review Board approved the study protocol.

\section{Results}

A single fluorescein angiographic diagnosis was made on the consensus of the two retinal specialists for each eye. These were as follows: (a) dry macular degeneration (drusen, RPE hyperplasia, focal atrophy, geographic atrophy): 33/74 (44.6\%); (b) occult choroidal neovascularization: $23 / 74(31.1 \%)$; (c) pigment epithelial detachment (serous): 6/74 (8.1\%); (d) minimally classic choroidal neovascularization: 5/74 (6.8\%); (e) normal angiogram: $4 / 74(5.4 \%)$; and (f) predominantly classic choroidal neovascularization: 3/74 (4.1\%).

The sampling method employed makes these percentages an estimate of the actual prevalence of AMD categories encountered in our ophthalmic photography department. However, these diagnoses are of both eyes for each subject and, as such, include a preponderance of dry AMD diagnoses.

Based on the concensus of the two retinal specialists, $54 \%$ (43) of eyes displayed no evidence of neovascular AMD, while for $46 \%$ (31) neovascular AMD was present. Kappa statistics for the two readers' diagnoses of neovascular AMD were excellent: nonstereo images 0.92 (95\% CI: 0.83-1.000), stereo pairs 0.87 (95\% CI: 0.75-0.98), stereo pairs with visual acuity and presenting complaint data 0.86 (95\% CI: 0.75-0.98).

Table 1 presents the raw data for the readers' evaluations of whether the colour images demonstrated neovascular AMD. Sensitivities and specificities for these evaluations were, in general, quite high. The highest sensitivities were noted for the stereo images combined with clinical data (1.00); however, in this setting, the specificity was relatively poor at 0.77 (95\% CI: 0.68-0.86). A slightly lower sensitivity, 0.98 (95\% CI: 0.96-1.00), but higher specificity, 0.83, (95\% CI: 0.75-0.91) was noted with the stereo pairs alone, and this trend was again repeated for the non-stereo, single retinal image evaluation (lowest sensitivity 0.95 (95\% CI: 0.90-1.00) and highest specificity 0.90 (95\% CI: 0.84-0.97)). In general, specificity was lost with the use of stereo images and history/acuity information, despite the fact that sensitivities rose in the setting of this additional information.

During the reading process of the colour images, the readers were also asked to document the particular clinical features that suggested the likelihood of an CNV to them. Subretinal fluid (SRF), subretinal lipid (SRL), and subretinal haemorrhage (SRH) were identified as the main features on the colour images that led to a suspicion of a choroidal neovascular membrane. The actual visualization of a pigmented membrane, vascular complex, sub-RPE haemorrhage, preretinal haemorrhage, or chorioretinal anastomosis was an uncommon finding (reported only once each and always in conjunction with subretinal fluid, haemorrhage, or lipid).

The sensitivity for each of the three most common clinical features, for identifying choroidal neovascularization on angiography, is presented in Table 2. The sensitivity and specificity of SRH, SRL, and SRF as predictors of the need for angiography were as follows:

$$
\begin{aligned}
& \text { SRH-0.81 (95\% CI: 0.68-0.95), } 0.95 \text { (95\% CI: 0.89-1.00) } \\
& \text { SRL-0.34 (95\% CI: 0.18-0.51), } 0.98 \text { (95\% CI: 0.93-1.00) } \\
& \text { SRF-0.56 (95\% CI: 0.39-0.73), } 0.95 \text { (95\% CI: 0.89-0.99) }
\end{aligned}
$$

For all three clinical features combined, the sensitivity was 0.94 (95\% CI: $0.85-1.00)$ and the sensitivity was 0.90 (95\% CI: 0.82-0.99).

Two (2/74) false negatives were recorded (by both observers for the same eye), but only on the nonstereo image evaluation. False-positives were noted by both observers in 5/74 eyes. For these five eyes, a falsepositive reading was recorded for all three-image evaluation methods: nonstereo, stereo, and stereo with history/acuity.

False positives occurred when pigment and drusen were misidentified as haemorrhage and lipid, respectively, in the setting of dry macular degeneration. Two cases in which SRH was present in the absence of an identifiable choroidal neovascular membrane were also encountered during this study, as were two eyes where SRF was the only clinical finding indicative of 
Table 1 Colour photograph interpretations $v s$ angiographic findings

(a) Nonstereo image evaluation

Angiographic findings

Colour image evaluation by readers $A / B$

$$
\text { Neovascular AMD absent }{ }^{a}
$$

$36 / 38$

$1 / 2$

Neovascular AMD present

Neovascular AMD present on angiogram ${ }^{\mathrm{b}}$

Neovascular AMD absent on angiogram

Kappa statistic: 0.92 (95\% CI: 0.83-1.000)

(b) Stereo image evaluation

\begin{tabular}{lcc}
\hline Angiographic findings & \multicolumn{2}{c}{ Stereo colour image evaluation by readers A/B } \\
\cline { 2 - 3 } & Neovascular AMD Absent & Neovascular AMD Present \\
\hline Neovascular AMD present on angiogram & $33 / 35$ & $8 / 6$ \\
Neovascular AMD absent on angiogram & $0 / 1$ & $33 / 32$
\end{tabular}

Kappa Statistic: 0.87 (95\% CI: 0.75 to 0.98 )

c. Stereo image evaluation with visual acuity and symptoms data

\begin{tabular}{lcc}
\hline Angiographic findings & \multicolumn{2}{c}{ Stereo colour image and clinical information evaluation by readers A/B } \\
\cline { 2 - 3 } & Neovascular AMD absent & Neovascular AMD present \\
\hline Neovascular AMD present on angiogram & $31 / 32$ & $10 / 9$ \\
Neovascular AMD absent on angiogram & $0 / 0$ & $33 / 33$ \\
Kappa statistic: 0.86 (95\% CI: 0.75-0.98) & &
\end{tabular}

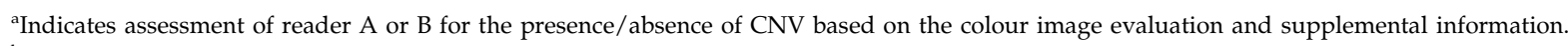

'Indicates the two readers' consensus for the presence/absence of CNV based on the evaluation of the fluorescein angiogram.

an underlying, active choroidal neovascular membrane.

In 47 eyes (63\%) recent macular symptoms ( $<4$ months) were described. Of the 74 eyes evaluated, 23 eyes (31\%) had symptoms in conjunction with a choroidal neovascular membrane on angiography, 24 (32\%) had symptoms and but no CNV on angiography, $16(22 \%)$ had no symptoms and no CNV on angiography, and 11 (15\%) had no symptoms despite the presence of an $\mathrm{CNV}$ on angiography (Table 3). A history of recent central vision loss was the symptom most likely to result in a recommendation for angiography by the readers in the absence of suspicious retinal pathology. In two cases, the readers recommended an IVFA on the basis of patient symptoms in the presence of only scattered drusen. In neither of these cases was an $\mathrm{CNV}$ present and in no case did the readers recommend an angiogram simply on the basis of poor visual acuity in the absence of concerning retinal findings.

\section{Discussion}

Colour fundus image evaluation using either stereo pairs or single images was adequate to identify a high percentage of subjects who demonstrated active neovascular AMD. False positives were most commonly associated with the misinterpretation of drusen and pigment clumps as lipid and SRH, respectively. When clinical information (symptoms and visual acuities) was given in conjunction with the fundus images, the highest sensitivity but lowest screening specificity was found.

On the basis of this information, we believe it is reasonable to consider colour fundus imaging to assist in the diagnosis and triage of patients with possible early exudative AMD. As part of the process of providing such a screening service, the decision to include clinical data or Amsler grid testing becomes pertinent. If the maximization of a screening program's sensitivity is desired, the inclusion of clinical data with stereo-pair images resulted in a $100 \%$ capture of individuals with active choroidal neovascularization. With this high capture rate, however, the increased rate of referral due to false positives $(77 \%$ specificity) would potentially hamper the cost-

effectiveness of a screening program. For this reason, the use of a single image or stereo pair may be adequate for screening. In general, giving the readers additional clinical information not only increased the false positive rate, but also increased the disagreement (decreased kappa) between observers. This is to be expected given 
Table 2 Clinical findings predictive of choroidal neovascularization on angiography

(a) Subretinal haemorrhage

\begin{tabular}{lcc}
\hline & $\begin{array}{c}\text { Subretinal } \\
\text { hamorrhage }^{\text {absent }}\end{array}$ & $\begin{array}{c}\text { Subretinal } \\
\text { haemorrhage } \\
\text { present }\end{array}$ \\
\hline CNV Absent $^{\mathrm{b}}$ & 40 & 2 \\
$\mathrm{CNV}^{\mathrm{C}}$ Present & 6 & 26
\end{tabular}

(b) Subretinal lipid

\begin{tabular}{|c|c|c|}
\hline & $\begin{array}{c}\text { Sub-retinal } \\
\quad \text { lipid } \\
\text { absent }^{\mathrm{a}}\end{array}$ & $\begin{array}{c}\text { Subretinal } \\
\text { lipid } \\
\text { present }\end{array}$ \\
\hline CNV absent ${ }^{\mathrm{b}}$ & 41 & 1 \\
\hline $\mathrm{CNV}$ present & 21 & 11 \\
\hline
\end{tabular}

(c) Subretinal fluid

\begin{tabular}{|c|c|c|}
\hline & $\begin{array}{c}\text { Subretinal } \\
\text { fluid } \\
\text { absent }^{\mathrm{a}}\end{array}$ & $\begin{array}{c}\text { Subretinal } \\
\text { fluid } \\
\text { present }\end{array}$ \\
\hline $\mathrm{CNV}_{\text {absent }}{ }^{\mathrm{b}}$ & 40 & 2 \\
\hline CNV present & 14 & 18 \\
\hline
\end{tabular}

(d) Subretinal haemorrhage or subretinal lipid or subretinal fluid

\begin{tabular}{lcc}
\hline & $\begin{array}{c}\text { None of three } \\
\text { characteristics } \\
\text { presenta }^{\mathrm{a}}\end{array}$ & $\begin{array}{c}\text { One of three } \\
\text { characteristics } \\
\text { present }\end{array}$ \\
\hline $\mathrm{CNV}^{\mathrm{C} \text { absent }} \mathrm{b}^{\mathrm{b}}$ & 38 & 4 \\
$\mathrm{CNV}$ present & 2 & 30 \\
\hline
\end{tabular}

${ }^{a}$ Indicates grading of reader A or B for the presence/absence of CNV based on the clinical finding noted.

'Indicates the two readers' consensus for the presence/absence of CNV based on the fluorescein angiogram.

Table 3 Contingency table evaluation of clinical features and macular symptoms

\begin{tabular}{lcc}
\hline & $\begin{array}{c}\text { No choroidal } \\
\text { neovascular } \\
\text { membrane }\end{array}$ & $\begin{array}{c}\text { Choroidal } \\
\text { neovascular } \\
\text { membrane }\end{array}$ \\
\hline No macular symptoms & $16(22 \%)$ & $11(15 \%)$ \\
Macular symptoms & $24(32 \%)$ & $23(31 \%)$ \\
\hline
\end{tabular}

Macular symptoms and the presence/absence of choroidal neovascularization on angiography.

the consequent increase in variability accompanying an increased error rate.

Amsler grid testing is a secondary tool that has been considered as a screening test for AMD. However, there is research to suggest that Amsler grid testing is of low sensitivity for choroidal neovascularization and does not correlate well with underlying ocular disease.${ }^{15}$ For this reason, difficulties with test administration, and the high sensitivities with imaging alone, the inclusion of Amsler grid data as part of a photographic AMD screening evaluation is questionable.

It is important to note that data from this study are not based on a population sample and, as such, simply generates sensitivities and specificities for the presence/ absence of neovascular AMD. To arrive at positive predictive values from these findings, AMD prevalence data are necessary for the particular population one wishes to study. Our decision to use a sample with a high prevalence of AMD does not invalidate the sensitivity and specificity data presented. In fact, the choice of a group of subjects with a high prevalence of neovascular AMD was essential to allow for meaningful confidence intervals for the sensitivity and specificity estimates. The use of a population-based methodology to evaluate photographic screening for neovascular AMD in a similar fashion would require thousands of subjects - given the fact that even in the highest risk populations, the prevalence of new-onset (active) exudative macular degeneration is likely to be extremely low. For example, estimates of AMD prevalence in geriatric nursing homes range from 24.5 to $30 \%,{ }^{16,17}$ however, only $10-12 \%$ of the AMD was neovascular, most of which was unlikely to be acute and treatable.

Although these data suggest that photographic screening is unlikely to be cost-effective if implemented in the general population, it may be feasible in higher risk groups (eg nursing home patients, outpatient cardiovascular clinic, etc). Alternatively, individuals who had already presented to their primary eye care provider with macular symptoms would comprise the population of interest if screening for angiographic triage was sought. Conceivably, imaging centres could be established in regions where ophthalmic care was absent or lacking. Images would then be forwarded to retina specialists for interpretation, after which the appropriate referral of high-risk individuals could be arranged.

Several previous publications have looked at the utility of fundus images for the identification of various features of macular degeneration. ${ }^{18,19}$ These systems have been well validated and exhibit good inter-rater reliability and reproducibility; however, many of these reports have focused on non-neovascular lesions of macular degeneration. The Age-Related Eye Disease (AREDS) study reported good to excellent inter-rater reliability and reproducibility using colour photography to identify characteristics of advanced AMD. ${ }^{20}$ However, the focus of the AREDS study on vitamin therapy precluded the comparison of photographs to fluorescein angiograms. In contrast, by comparing photographic grading to the definitive standard of fluorescein angiography, our study 
was able to identify the sensitivity and specificity of lesion characteristics on colour photographs that indicate an active neovascular complex that requires urgent referral for angiography. Furthermore, the population evaluated in the current study consisted of patients recently referred for angiography because of a new retinal findings or a change in their vision. Our study therefore demonstrates the potential utility of photographic screening in patients with recent neovascular change; that is, in the patients who are most likely to benefit from appropriate triage for photodynamic therapy or ablative laser.

\section{References}

1 Hennekens CH, Buring J. Epidemiology in Medicine. Little Brown and Company: Boston/Toronto, 1987.

2 Singer D, Nathan D, Fogel H, Schachat A. Screening for diabetic retinopathy. Ann Intern Med 1992; 116: 660-671.

3 Javitt J, Canner J, Sommer A. Cost effectiveness of current approaches to the control of retinopathy in type 1 diabetics. Ophthalmology 1989; 96: 255-264.

4 Javitt J, Aiello L. Cost-effectiveness of detecting and treating diabetic retinopathy. Ann Intern Med 1996; 124: 164-169.

5 Sussman E, Tsiaras G, Soper K. Diagnosis of diabetic eye disease. JAMA 1982; 247: 3231-3234.

6 Kinyoun J, Barton F, Fisher M, Hubbard L, Aiello L, Ferris F. Detection of diabetic macular edema. Ophthalmoscopy versus photography - Early Treatment Diabetic Retinopathy Study Report Number 5. The ETDRS Study Group. Ophthalmology 1989; 96: 647-651.

7 Moss SE, Klein R, Kessler SD, Richie KA. Comparison between ophthalmoscopy and fundus photography in determining severity of diabetic retinopathy. Ophthalmology 1985; 92: 62-67.

8 Diabetic Retinopathy Study Research Group. Photocoagulation of proliferative diabetic retinopathy. DRS Report. Ophthalmology 1981; 88: 583-600.

9 Early Treatment Diabetic Retinopathy Study. Photocoagulation for diabetic macular edema. Report. Arch Ophthalmol 1985; 26: 983-991.
10 Treatment of Age-Related Macular Degeneration with Photodynamic Therapy (TAP) Study Group. Photodynamic therapy of subfoveal choroidal neovascularization in agerelated macuar degeneration with verteporfin. One year results of 2 randomized clinical trials-Tap Report 1. Arch Ophthalmol 1999; 117: 1329-1345.

11 The Age-Related Eye Disease Study Research Group. A randomized placebo-controlled, clinical trial of high dose supplementation with vitamins $\mathrm{C}$ and $\mathrm{E}$ and beta carotene for age-related macular degeneration and vision loss. AREDS Report no. 8. Arch Ophthalmol 2001; 119: 1417-1436.

12 The Age-Related Eye Disease Study Research Group. The age-related eye disease study system for classiflying agerelated macular degeneration from stereoscopic colour fundus photographs: The Age-Related Eye Disease Study Report Number 6. Am J Ophthalmol 2001; 132: 668-681.

13 Guidelines for using verteporfin (visudyne) in photodynamic therapy to treat choroidal neovascularization due to age-related macular degeneration and other causes. Retina 2002; 26: 6-18.

14 Haddad WM, Seres A, Coscas G, Soubrane G. Presentation delay in patients affected with age-related macular degeneration. Graefe's Arch Clin Exp Ophthalmol 2002; 240(1): 31-34.

15 Ariyasu RG, Lee PP, Linton KP, La Bree LD, Azen SP, Siu AL. Sensitivity, specificity, and predictive values of screening tests for eye conditions in a clinic-based population. Ophthalmology 1996; 103(11): 1751-1760.

16 Whitmore WG. Eye disease in a geriatric neursing home population. Ophthalmology 1989; 96: 393-398.

17 Kiess RD, Kollarits CR, Mehelas TJ, Slak S, Burns RJ, Todia WJ. Visual loss in geriatric residents of northwestern Ohio nursing homes. Ohio State Med J 1984; 80: 235-237.

18 Klein R, Davis MD, Magli YL, Segal P, Klein BEK, Hubbard L. The Wisconsin age-related maculopathy grading system. Ophthalmology 1991; 98: 1128-1134.

19 Bressler NM, Bressler SB, West SK, Fine SL, Taylor HR. The grading and prevalence of macular degeneration in Chesapeake Bay watermen. Arch Ophthalmol 1989; 107: 847-852.

20 The Age-Related Eye Disease Study Group. The age-related eye disease study system for classifying age-related macular degeneration from stereoscopic colour fundus photographs: The Age-Related Eye Disease Study Report Number 6. Arch Ophthalmol 2001; 131: 167-175. 